\title{
Estudio de la situación socioeconómica y académica del docente de la Facultad de Medicina de la Universidad Peruana Cayetano Heredia.
}

Study of the social, economic and academic situation of the professor of the Faculty of Medicine in the Universidad Peruna Cayetano Heredia.

PAMO REYNA Oscar*, RUIZ DIAZ Jorge E**

*Profesor Asociado, Departamento de Medicina, Universidad Peruana Cayetano Heredia.

** Bachiller en Medicina, Universidad Peruana Cayetano Heredia.

\section{SUMMARY}

One hundred and twenty one teachers, $51.3 \%$ of the total of the faculty of medicine Alberto Hurtado of UPCH were surveyed in order to determine some social, economic and academia characteristics. We found: age average, $49.4 \mathrm{yr} ; 89.3 \%$ male; $87.6 \%$ were married; $85.8 \%$ lived in middle and high social level areas; average of children $2.8 ; 8.3 \%$ were full-time teachers; average of years in teaching $19.5 \mathrm{yr} ; \mathbf{9 5 . 9 \%}$ were attracted for teaching; $85.1 \%$ would not abandon teaching; $\mathbf{4 2 . 4 \%}$ were satisfied; $52.9 \%$ had training in teaching methods; $54.5 \%$ were doing research; $\mathbf{5 5 . 9 \%}$ had published; $\mathbf{9 0 . 0 \%}$ belong to the Ministry of Health-Hospitals; $79.2 \%$ do private practice; $75.0 \%$ own a house; $52.5 \%$ had other member of their family working and $72.5 \%$ had private practice as the main income. We concluded that our teaching staff belongs in average, to the middle class, do teaching as an extra activity, have interest and experience in teaching but do not have enough pedagogical education and that research and publications could increase.

KEY WORDS: Professor of medicine, socioeconomic situation, academic situation, education.

\section{RESUMEN}

Una muestra de 121 docentes, 51.3\% del total, de la Facultad de Medicina Alberto Hurtado de la UPCH fue encuestada con el objeto de conocer sus características sociales, económicas y académicas. Hallamos: promedio de edad: 49.4 años; 89.3\% varones; $87.6 \%$ casados; $85.8 \%$ residían en distritos de nivel medio y alto; promedio de 2.8 hijos; $8.3 \%$ a dedicación exclusiva o tiempo completo; promedio de 19.5 años en la docencia; $95.9 \%$ hacían docencia por atracción; $85.1 \%$ no la dejarían; $42.4 \%$ se sentían satisfechos; $52.9 \%$ tenían capacitación en tecnología educativa; $54.5 \%$ investigaba; 55.9\% publicaba; $90.0 \%$ trabajaba para el Ministerio de Salud; 79.2\% hacía práctica 
privada; $75.0 \%$ tenía casa propia; $52.5 \%$ tenía familiares que generaban ingresos y $72.5 \%$ tenía la práctica privada como principal fuente de ingreso. Se concluye que nuestros docentes en promedio son de nivel socioeconómico medio, realizan la docencia como actividad complementaria, tienen vocación por la enseñanza y mucha experiencia, más no suficiente formación pedagógica y que podrían incrementar la investigación y publicaciones.

PALABRAS CLAVE: docente de medicina, aspecto económico, aspecto académico, docencia

\section{INTRODUCCION}

Desde su fundación en 1961 la Universidad Peruana Cayetano Heredia (UPCH) ha contribuido de manera muy importante al desarrollo de la Medicina Peruana. En el período 1964-1989, se titularon 1444 médico-cirujanos de la Facultad de Medicina Alberto Hurtado de esta universidad; y en el periodo 1965-1989 egresaron 891 especialistas de la Escuela de Postgrado Víctor Alzadora Castro (3). Es decir, nuestra Facultad de Medicina ha formado directamente al diez por ciento de los médicos en ejercicio actual. Sin embargo, su influencia es mucho mayor por el efecto multiplicador de la destacada labor de sus egresados en su afán de solucionar los problemas de salud que aquejan a nuestro país.

A pesar de los escasos estudios que se han hecho sobre docencia universitaria en el país, ésta tiene características comunes y otras peculiares dependiendo de si es privada o estatal y de la naturaleza de los estudios que abarca. Sobre docencia médica también son pocas las evaluaciones realizadas. El 36.0\% de los médicos de Lima Metropolitana (1) y el $16.1 \%$ de los médicos a nivel nacional (10) realizan docencia universitaria, mientras que el $41.0 \%$ desearía trabajar en una universidad (1). De estos médicos que realizan docencia, el $21.3 \%$ lo hace en entidades particulares, incluyendo nuestra universidad. La masa activa del profesorado de nuestra facultad es de 236 y sus problemas y aspiraciones son soslayados, no han sido cuantificados. En este contexto debemos mencionar la autoevaluación de las Facultades de Medicina del Perú realizado en 19851986 por la ASPEFAM (Asociación Peruana de Facultades de Medicina), a través de cuatro seminarios. Según ella "Los hallazgos de la evaluación realizada muestran un desarrollo modesto de la educación médica en el país" (2). Creemos que los problemas y dificultades que afronta cada Facultad de Medicina deben ser estudiados y detallados para comprender la preocupante conclusión de la ASPEFAM, tal que permita hacer correcciones y mejoras. Precisamente el presente estudio tiene por objeto dar a conocer las características sociales, económicas y académicas del docente de la Facultad de Medicina de nuestra universidad, lo que nos permitirá tomar las medidas adecuadas al respecto en cualquier proyecto de planificación futuro.

\section{MATERIAL Y METODOS}

Para cumplir con el objetivo trazado, se llevó a cabo un estudio descriptivo, prospectivo y transversal de tipo encuesta. 
La selección de la población a estudiar se realizó, teniendo como fuente el listado de docentes proporcionado por el Libro de Presupuesto de la Universidad Peruana Cayetano Heredia de agosto de 1989 (12).

Sólo se consideró a profesores ordinarios que tuvieran las siguientes cargas horarias: dedicación exclusiva (DE), tiempo completo (TC), tiempo parcial $20 \mathrm{hrs}$ (TP-20), tiempo parcial $10 \mathrm{hrs}$. (TP-10) y tiempo parcial $5 \mathrm{hrs}$.(TP-5); no considerándose los profesores con dedicación horaria y aquellos contratados.

En base a los datos contenidos en el Libro de Presupuesto de la UPCH de Agosto de 1989, se obtuvo un total de 236 profesores ordinarios de las diferentes categorías, de los cuales $4(1.69 \%)$ estaban ausentes.

De los 232 profesores ordinarios, 68 (29.3\%) son principales, 43 (18.5\%) son asociados y $121(52.2 \%$ son auxiliares.

Se realizó una encuesta piloto en 15 docentes que permitió dar forma final a la encuesta. El estudio se realizó entre los meses de abril a junio de 1990.

La encuesta incluía 40 items para los siguientes indicadores:

1.Datos generales y demográficos; edad, sexo, estado civil, distrito de domicilio, colegio de procedencia y composición familiar.

2.Laborales: profesión de los docentes, categoría docente, régimen de dedicación a la universidad, años dedicados a la docencia, tiempo de docencia en la UPCH, niveles de ejercicio de la docencia, labor docente en otras universidades, participación en el gobierno de la universidad.

3.Vocacionales: motivos por los que ingresó a la docencia, motivos por los que no deja la docencia, motivos por los que dejaría la docencia y sentimiento con relación al trabajo docente.

4.Académicos: capacitación en tecnología educativa, grados académicos, especialidad, conocimiento y/o dominio de otros idiomas, dedicación a la investigación, publicación de artículos y/o libros, suscripción a publicaciones científicas extranjeras, viajes académicos.

5.Condiciones materiales para el trabajo: apoyo secretarial, ambiente de trabajo docente, calidad y condición del material bibliográfico utilizado.

6.Socioeconómicos: instituciones en las que trabaja, práctica privada profesional, propiedades e inmuebles, viajes recreativos, otras fuentes de ingreso familiar, actividades económicas complementarias, principal fuente de ingresos.

Todos estos datos se recogieron a través de una encuesta anónima con preguntas cerradas y respuestas categorizadas.

La elección de los profesores que fueron encuestados se hizo al azar, manteniéndose la proporción de las diferentes categorías. La encuesta fue realizada de manera personal y fueron contestadas de inmediato en su mayoría, mientras que algunos la entregaron en una fecha acordada. 
Fueron entrevistados 127 docentes, de los cuales colaboraron 121 (51.3\% del total): principales $36(29.8 \%)$; asociados $28(23.1 \%)$ y $57(47.1 \%)$ auxiliares. Se negaron a responder 6 docentes; 3 principales, 1 asociado y 2 auxiliares.

\section{RELACION DE SIGLAS UTILIZADAS:}

ASPEFAM: Asociación Peruana de Facultades de Medicina

DE: Dedicación exclusiva

DES: Docencia en servicio

TC: Tiempo completo

TP-20: Tiempo Parcial 20 hrs.

TP-10: Tiempo Parcial 10 hrs.

TP-5: Tiempo Parcial 5 hrs.

UPCH:Universidad Peruana Cayetano Heredia

INS: Instituto Nacional de Salud

IPSS: Instituto Peruano de Seguridad Social

MSP: Ministerio de Salud Pública

\section{RESULTADOS}

\section{DATOS GENERALES Y DEMOGRAFICOS}

El promedio de edad de la población estudiada fue de 49.4 años (rango: 30-68); 104 docentes $(85.9 \%)$ tuvieron edades comprendidas entre los 30 y 59 años de edad y 17 $(14.1 \%)$ edades iguales o mayores de 60 años.

Por categorías, los auxiliares tuvieron un promedio de 45.6 años (rango: 30-63); 53 $(93.0 \%)$ con edades entre 30 y 59 años y $4(7.0 \%)$ igual o mayor a 60 años.

Los asociados tuvieron un promedio de 46.8 años (rango: 38-66); 27 (96.4\%) con edades entre los 38 y 59 años y 1 (3.6\%) igual o mayor a 60 años.

En los principales la edad promedio fue de 57.4 años (rango: 48-68); 24 (66.7\%) con edades entre 48 y 59 años y $12(33.3 \%)$ igual o mayor a 60 años.

En cuanto al sexo, 108 docentes (89.3\%) pertenecían al masculino y $13(10.7 \%)$ al femenino, $106(87.6 \%)$ de los docentes eran casados, 7 (5.8\%) solteros, $4(3.3 \%)$ viudos, $3(2.5 \%)$ convivientes y $1(0.8 \%)$ divorciado.

De 120 docentes que respondieron, 24 (20.0\%) domicilian en Santiago de Surco, 17 (14.2\%) en Miraflores, 13 (10.8\%) en San Borja, 12 (10.0\%) en San Isidro, 6 (5.0\%)en la Molina, Pueblo Libre y Cercado de Lima. Los restantes 36 docentes que representan el $25.0 \%$ del total se distribuyen en 11 distritos de la provincia de Lima y en la provincia constitucional del Callao.

De 120 docentes que respondieron, $70(58.3 \%)$ refirieron haber realizado estudios en colegios privados y $50(41.7 \%)$ en colegios estatales. 
Por categorías tenemos: 32 (56.1\%) docentes auxiliares procedían de colegios privados y $25(43.9 \%)$ de colegios estatales. De los docentes asociados, $16(57.1 \%)$ procedian de colegios privados y $12(42.9 \%)$ de colegios estatales. Por último $22(62.9 \%)$ de los docentes principales procedían de colegios privados y $13(36.1 \%)$ de colegios estatales.

De 120 docentes que respondieron, la composición familiar tuvo un promedio de 4.2 miembros. Por categorías, el promedio de personas por familia fue de 4.1 en los auxiliares, de 4.7 en los asociados y de 4.4 en los principales.

Hubo 108 docentes que refirieron tener uno o más hijos, siendo el promedio de 2.8 hijos. Los docentes auxiliares tuvieron un promedio de 2.5 hijos, los asociados de $2.9 \mathrm{y}$ los principales de 3.1 .

De los docentes que tenían hijos, 103 (95.4\%) señalaron tener uno o más de ellos estudiando. El número total de hijos estudiantes fue de 252, de los cuales 111 (44.1\%) eran universitarios, $120(47.6 \%)$ escolares y $21(8.3 \%)$ preescolares.

\section{LABORALES}

$120(98.2 \%)$ indicaron ser médico-cirujanos de profesión y $1(0.8 \%)$ era biólogo. Ninguno de los docentes refirió tener otra profesión. De los 121 docentes, 36 (28.8\%) eran principales, $28(23.1 \%)$ asociados y $57(47.1 \%)$ auxiliares. Ellos representan el $51.9 \%, 65.1 \%$ y el $47.1 \%$ del total de las categorías a la cual pertenecen respectivamente.

De los 121 docentes: 7 (5.8\%) tenían DE, 5 de ellos como DES; 3 (2.5\%) trabajan a TC; $82(67.8 \%)$ trabajan a TP-20 hras., 45 de ellos como DES; 25 (20.6\%) trabajan a TP-10 hras. Y $4(3.3 \%)$ trabajaban a TP-5 hrs.

En 120 docentes que contestaron la pregunta, el promedio de años dedicados a la docencia fue de 19.5 años (rango: 3-39); 90 docentes (75.0\%) refirieron tener más de 10 años de ejercicio de labor docente y 30 (25.0\%) menos de 10 años. Por categorías, en cuanto a los años dedicados a la docencia, tenemos: los auxiliares con un promedio de 14.5 años (rango: 3-38); los asociados de 17.3 años (rango: 9-34) y los principales de 29.2 años (rango: 20-39).

De 120 docentes que respondieron, el promedio de años como docente en la UPCH fue de 17 años (rango: 3-29); 87 (72.5\%) refirieron más de 10 años ejerciendo y 33 (27.5\%) menos de 10 años. Por categorías tenemos: los auxiliares con un promedio de 12.6 años (rango: 3-29), los asociados de 15.8 años (rango: 4-29) y los principales de 25.1 años (rango: 13-29).

Cuarenta y tres docentes (35.5\%) realizaban labor a nivel preclínico; $110(90.9 \%)$ a nivel clínico; $86(71.1 \%)$ a nivel de prácticas preprofesionales y $100(82.6 \%)$ a nivel de residentes y escuela de postgrado.

Nueve docentes $(7.4 \%)$ refirieron realizar labor docente en un solo nivel; 17 (14.1\%) en dos niveles y $95(78.5 \%)$ en tres o más niveles. 
Veintisiete $(22.3 \%)$ de los docentes refirieron realizar labor docente en otros centros universitarios: $6(22.2 \%)$ señalaron hacerlo sólo como invitados y $21(77.8 \%))$ en forma regular ( 8 de ellos en otras universidades particulares y 13 en universidades estatales).

De 120 docentes que respondieron la pregunta, $55(45.8 \%)$ refirieron participar en alguna instancia de gobierno de la universidad y 65 (54.2\%) no.

\section{VOCACIONALES}

Ciento dieciseis docentes $(95.9 \%)$ indicaron que ingresaron a la docencia porque sentían atracción por ella; $17(14.0 \%)$ por el prestigio que tiene el ser docente de la universidad; $5(4.1 \%)$ por considerarlo un ingreso extra; $1(0.8 \%)$ porque no tenía trabajo. 14 (11.6\%) consideraron otros motivos ( 7 como una forma de mantenerse actualizado en los conocimientos de la profesión; 4 por convenio suscrito entre la universidad y el Ministerio de Salud; 1 por vocación de servicio; 1 como gratitud hacia el Alma Mater; y 1 porque quería conocer la docencia en nuestro medio).

Ciento tres $(85.1 \%)$ de los profesores indicaron que no abandonan la docencia por el beneficio de la enseñanza e investigación; 23 (19.0\%) por tener un ambiente de trabajo adecuado; $21(17.4 \%)$ por la flexibilidad en el horario; $6(4.9 \%)$ porque representaba un ingreso extra; 11 docentes $(9.1 \%)$ consideraron otros motivos (7 por ser un estímulo para mantenerse actualizado en su profesión; 2 por ser una satisfacción personal; 1 por considerarlo como una vocación de servicio y 1 por gratitud hacia el Alma Mater).

De 107 docentes que respondieron, sobre los motivos por los que dejaría la docencia, 46 $(42.4 \%)$ señalaron que lo harían por no contar con las facilidades adecuadas para el ejercicio de la labor docente; 38 (35.5) porque le restaba tiempo para el ejercicio de otras actividades que ellos consideraban prioritarias; $15(14.1 \%)$ porque habían perdido interés en la docencia; 5 (4.7\%) señalaron otros motivos tales como la lejanía de la sede docente, incapacidad personal, ausencia de planificación universitaria, etc. Un total de 7 docentes $(6.5 \%)$ señalaron que no dejarían la docencia por ningún motivo.

De 118 docentes que respondieron, sobre el sentimiento con relación al trabajo docente, $50(42.4 \%)$ refirieron sentirse satisfechos; 68 (57.6\%) se sentían insatisfechos. De ellos, $11(16.2 \%)$ se sentían frustrados.

De los que se sentían insatisfechos, según categorías tenemos: auxiliares 34 (60.7\%).asociados $16(59.3 \%)$ y principales $18(51.4 \%)$.

\section{ACADEMICOS}

Sesenta y cuatro $(52.9 \%)$ de los docentes refirieron haber tenido una o más participaciones en cursos, seminarios o talleres sobre pedagogía médica. 57 docentes (47.1\%) no lo habían hecho.

Treinta y ocho (31.4\%) eran bachilleres; 28 (23.1\%) maestros y 55 (45.5\%) doctores.

Ciento catorce docentes $(94.2 \%)$ refirieron tener una o más especialidades (4 de ellos en forma no escolarizada); 7 (5.8\%) no tenían especialización, de los cuales 5 tenían maestría y 2 sólo bachillerato. 
Ciento diecinueve docentes $(98.3 \%)$ manifestaron tener conocimiento y/o dominio de uno o más idiomas diferentes al nuestro; y sólo $2(1.7 \%)$ indicaron no tenerlo, 117 $(98.3 \%)$ refirieron tener conocimiento y/o dominio de inglés, 26 (21.8\%) de francés, 19 $(15.9 \%)$ de portugués, $1(0.8 \%)$ de japonés y $1(0.8 \%)$ de ruso.

Setenta y cuatro docentes $(62.2 \%)$ refirieron conocer y/o dominar un idioma aparte del nuestro, $34(28.6 \%)$ dos idiomas y $11(9.2 \%)$ tres o más idiomas.

Sesenta y seis docentes $(54.5 \%)$ refirieron dedicarse a la investigación en forma permanente y $55(44.5 \%)$ indicaron no hacerlo.

Según categorías tenemos: En los auxiliares 29 (50.9\%) lo hacían y 28 (49.1\%) no lo hacían. En los asociados 14 (50.0\%) investigación y 14 (50.0\%) no investigaban. En los principales $23(63.9 \%)$ lo hacían y $13(36.1 \%)$ no realizaban investigación alguna.

Por grados académicos tenemos: En los bachilleres 16 (42.1\%) investigaban y 22 (57.9\%) no lo hacían. En los maestros 18 (64.3\%) realizaban investigación y $10(35.7 \%)$ no realizaban investigación alguna. En los doctores 32 (54.5\%) lo hacían y 23 (41.8\%) no lo hacían.

En general de los 66 docentes que refirieron realizar investigación en forma permanente, $44(66.7 \%)$ habían publicado uno o más trabajos en los últimos dos años. Mientras que de los 55 docentes que indicaron no realizar investigación 17 (30.9\%) habían publicado uno o más trabajos en los últimos dos años.

Ciento nueve docentes contestaron la pregunta sobre la publicación de artículos en revistas y/o libros en los últimos dos años; 61 (55.9\%) precisaron una o más publicaciones y 48 (44.1\%) refirieron no haber publicado trabajo alguno. De aquellos que publicaron, el promedio fue de 0.8 publicaciones por semestre.

Según categorías, de los que tenían una o más publicaciones, tenemos: auxiliares 23 $(46.9 \%)$ con un promedio de 0.7 por semestre; asociados $17(60.7 \%)$ con un promedio de 1 por semestre y principales $21(65.6 \%)$ con un promedio de 0.7 por semestre. De aquellos que no habían publicado tenemos: auxiliares 26 (53.1\%) asociados: 11 (39.3\%) y principales $11(34.4 \%)$.

Por grados académicos, de los que tenían una o más publicaciones, tenemos bachilleres $13(40.6 \%)$ con un promedio de 0.5 por semestre: maestros $17(63.0 \%)$ con un promedio de 1.1 por semestre; doctores $31(62.0 \%)$ con un promedio de 0.7 por semestre. De aquellos que no habían publicado tenemos: bachilleres19 (50.4\%); maestros 10 (37.0\%) y doctores $19(38.0 \%)$.

Cuarenta y seis docentes (38.1\%) refirieron estar suscritos a una o más publicaciones científicas extranjeras; no así 75 (61.9\%).

De 120 docentes que respondieron sobre viajes académicos al exterior en los últimos dos años, 47 (39.2\%) refirieron haber realizado uno o más; no así 73 (60.8\%).

\section{CONDICIONES MATERIALES PARA EL TRABAJO DOCENTE}


Respondieron 49 (40.5\%) docentes que tenían apoyo secretarial y $72 \quad(59.5 \%)$, que no lo tenían.

Respondieron afirmativamente el tenerlo, sobre ambiente de trabajo docente (oficina, escritorio, etc.) 58 (47.9\%); y 63 (52.1\%) refirieron no contar con dicha infraestructura.

Ciento siete docentes $(88.3 \%)$, señalaron que utilizaban material bibliográfico de su propiedad; 66 (54.5\%), hacían uso de material proveniente de la Biblioteca Central de la Universidad; 27 (22.3\%) utilizaban material perteneciente al Departamento Académico al que pertenecían y $34(28.1 \%)$, señalaron que utilizaban material procedente de otras bibliotecas universitarias, de institutos, entidades particulares, etc.

\section{SOCIOECONOMICOS}

De 120 docentes que respondieron sobre instituciones en las que trabaja, $108(90.0 \%)$ trabajaban para el Ministerio de Salud; 49 (40.8\%) en una entidad privada; 21 (17.5\%) realizaban labor docente en otras universidades; 7 (5.8\%) docencia sólo en la UPCH; 3 $(2.5 \%)$ trabajaban en la Sanidad de las Fuerzas Armadas; $2(1.7 \%)$ en el IPSS y 1 $(0.8 \%)$ en el INS.

Respondieron afirmativamente hacer práctica privada profesional, 95 docentes $(79.2 \%)$, mientras que 25 (20.8\%) refirieron no hacerlo.

De 120 docentes, $90(75.0 \%)$ señalaron tener vivienda propia; 18 (15.0\%) vivienda alquilada; $3(2.5 \%)$ en alquiler venta; y, de $9(7.5 \%)$ era propiedad de sus familiares o del cónyuge.

Referente a la tenencia de consultorio, de 120 que respondieron, $96(80.0 \%)$ lo hicieron afirmativamente y $24(20.0 \%)$ negativamente. De los que tenían consultorios; 48 $(50.0 \%)$ refirieron que eran alquilados; $45(46.9 \%)$ de su propiedad; y, $1(1.1 \%)$ en préstamo.

De 120 docentes que respondieron, sobre la tenencia de carro propio; 104 (86.7\%) eran propietarios y $16(13.3 \%)$ no contaban con ello.

Setenta docentes (58.3\%) eran miembros de algún Club Social-Recreativo-Deportivo y $50(41.7 \%)$ no lo eran.

Veintiun docentes (17.5\%) eran accionistas de alguna clínica y 99 (88.5\%) refirieron que no.

De 118 docentes que respondieron, 45 (38.1\%) indicaron haber realizado uno o más viajes recreativos al exterior en los últimos dos años y 73 (61.9\%) no lo hicieron.

De 120 docentes que respondieron, 63 (52.5\%) refirieron tener uno o más miembros que generaban ingreso familiar y $57(47.5 \%)$ no tenían.

Por categorías en cuanto a los que tenían algún miembro más en la familia que generaba ingreso, tenemos: auxiliares $31(54.4 \%)$; asociados $18(64.3 \%)$ y principales $21(60.0 \%)$. 
Y, de aquellos que no tenían, tenemos: auxiliares 26 (45.6\%); asociados, 10 (35.7\%) y principales $14(40.0 \%)$.

De 120 docentes que respondieron; 4 (3.3\%) refirieron participar en alguna actividad económica no relacionada a su profesión; 116 (96.7\%) no lo hacían.

De 120 docentes que respondieron, 87 (72.5\%) señalaron que la principal fuente de ingresos era proveniente de su actividad privada profesional, $24(20.0 \%)$ de su trabajo como médico de hospital, 7 (5.8\%) de su trabajo como docente universitario y $2(1.7 \%)$ de fuentes no vinculadas a su labor como docente o profesional.

\section{DISCUSION}

Como podemos apreciar el promedio de edad de nuestros docentes es de 49.4 años, asociados 46.8 años, principales 57.4 años. Similar al encontrado por Alarcón y col., 46.1 años, en una población de docentes médicos de universidades particulares (1) y es mayor que el promedio de edad de los docentes de otras carreras, como lo hallado por Dejo, 40.8 años, en un estudio sobre los docentes universitarios de Lima Metropolitana (5). Esto puede explicarse por el tiempo que toma la formación de un médico y su especialización antes de ser incorporado a la docencia. Inclusive el promedio de edad del docente de medicina es mayor que el del médico en general, 44.0 años (10).

Existe como en otras actividades de la carrera universitaria predominio del sexo masculino. Esto es bien notorio en nuestra Facultad donde la proporción es de 9.1, mayor que la proporción que existe en el docente universitario en general que es de 4.1 (5). Si consideramos que la proporción de varones a mujeres en los egresados de Medicina de la UPCH, que es la principal fuente de provisión de nuevos profesores, es de 4 a 1, la reducción es importante quizás por la alta competitividad entre otros factores no estudiados. La gran mayoría de nuestros docentes, 87.6\%, eran casados. Esta proporción puede variar de acuerdo al tipo de sociedad, por ejemplo sólo el $60.9 \%$ de los docentes de medicina de Cochabamba eran casados (7).

El $85.8 \%$ de nuestros docentes residen en distritos considerados como de estrato socioeconómico medio y alto, los cuales geográficamente son los más distantes de la sede universitaria y de las sedes docentes.

Los profesores principales proceden en mayor proporción de colegios privados lo que probablemente refleja una situación de status socioeconómico de la década del 50 y que ha cambiado en las décadas siguientes por el mayor acceso a la educación médica, tal que la proporción de docentes con procedencia de colegios estatales se incremente. Con esta misma idea, podemos predecir que debido al deterioro de la enseñanza en los colegios estatales en los últimos años se incrementará el número de docentes procedentes de colegios privados. Lo hallado en este estudio es menor a lo encontrado en el estudio de Cochabamba, 60.7\% (7).

En cuanto a composición familiar, el promedio de 4.2 personas y el promedio de 2.8 hijos por familia de los docentes refleja la composición de una familia de estrato socioeconómico medio de nuestra sociedad (6). 
Existe un alto porcentaje, 95.4\%, de los docentes con hijos estudiantes los cuales son dependientes económicamente por su naturaleza.

La mayoría de nuestros docentes, $91.7 \%$ tiene regímenes a dedicación parcial horaria. Lo ideal sería que la universidad cuente con una mayor proporción de docentes a tiempo completa, lo cual permitiría no sólo un mejor desarrollo académico sino que facilitaría una mayor dedicación a la investigación. Esta necesidad del docente universitario a exclusividad en la universidad peruana ya ha sido enunciada antaño (4).

Nuestros docentes cuentan con experiencia considerable en el ejercicio de su labor, en general $96.7 \%$ con más de 5 años ejerciendo y un promedio de 19.5 años. Específicamente en cuanto al tiempo de docencia en nuestra universidad, tenemos un $90.8 \%$ de docentes con más de 5 años ejerciendo y un promedio de 17 años.

Considerando que la UPCH tiene 29 años de fundada existe una proporción importante de profesores dedicados a la docencia con un tiempo igual o mayor a 30 años, 21 profesores $(17.5 \%$ del total). De éstos, la mayoría son de la categoría principales, 18 profesores $(85.7 \%)$.

La enseñanza a nivel preclínico y básico se realiza generalmente en el local de la universidad. Los demás niveles se realizan en los centros hospitalarios lo cual facilita que los profesores enseñen en varios niveles, teniendo un $78.5 \%$ de ellos ejerciendo en tres o más niveles.

Aproximadamente la quinta parte de nuestros docentes también realizan labor docente en otros centros universitarios debido a que es una fuente extra de ingresos, no obstante que la UPCH recomienda a sus profesores cierta exclusividad (13). Esto ocurre usualmente en las sedes docentes-hospitalarias donde hay concurrencia de varias universidades no así en el Hospital Cayetano Heredia.

Existe un porcentaje importante de docentes que participan en el gobierno de la universidad (45.8\%), lo cual creemos es beneficioso.

A pesar que el $95.9 \%$ de los profesores refirieron sentir atracción por la docencia y $85.1 \%$ no la dejaría por la enseñanza e investigación que realizan, el 57.6\% señalaron sentirse insatisfechos de su trabajo como docente, porque consideraban que no tenían facilidades (42.4\%), y, que les pagaban poco (35.5\%). De aquellos insatisfechos, 11 profesores $(16.1 \%)$ manifestaron sentirse frustrados.

Llama la atención el alto porcentaje de profesores que no han tenido capacitación en pedagogía médica. La mayoría de aquellos que tuvieron alguna formación pedagógica lo hicieron por cuenta propia y generalmente a través de seminarios y cursos cortos los cuales son considerados como insuficientes (8). La falta de preparación pedagógica de los que ejercen la docencia en las universidades, desde los primeros años de estudios o ciclo básico, dificulta el aprendizaje notablemente (9).

Es importante hacer notar que entre los profesores de la categoría principal es decir entre los de mayor antigüedad, existe una mayor proporción que han obtenido el grado de doctor sin haber optado previamente el de maestro merced a reglamentos transitorios que les permitieron regularizar su situación ya que la actual ley universitaria $\mathrm{N}^{\mathrm{o}} 23733$ exige antes el grado de maestro. En general tenemos que el $68.6 \%$ de nuestros docentes 
tienen otro grado académico aparte del bachillerato. La especialización en la profesión médica es prácticamente una exigencia en la carrera docente, así el 94.6\% de nuestros docentes tenían una o más especialidades y el $96.5 \%$ eran escolarizados. Esta proporción es mayor que la de los docentes médicos especialistas de Lima Metropolitana general, 85.6\% (1). Con respecto al especialista y docencia, según Vasco Uribe: "Que llegan a ella (la universidad) como su única fuente de trabajo al menos en un principio, lo que los coloca en contacto más directo con la institución, los hace participar de su organización y los hace depender de ella más íntimamente, aunque estén allí solo para adquirir prestigio y aspiren a trabajar particularmente"(14); lo que encierra cierta verdad.

La gran mayoría de los profesores, $98.3 \%$, conocía y/o dominaba uno o más idiomas diferentes al nuestro, siendo el inglés el más importante, $98.3 \%$ debido a la influencia de la medicina norteamericana en los últimos cincuenta años. La proporción de docentes que no realizan investigación en forma permanente es muy alta, $45.5 \%$, lo cual es contrario a lo recomendado y exigido en la universidad contemporánea. Debemos considerar que "Dentro del claustro universitario, la labor de un profesor es triple: docencia, investigación y capacitación de futuros profesionales" y "Quien investiga, es decir el hombre activo en el trabajo intelectual resulta ser el más capacitado para enseñar. "Debido a su vocación y hábitos profesionales, suele proporcionar nuevos planteamientos e impartir una enseñanza que induce al estudiante a desarrollar su espíritu de iniciativa para enfrentar novísimas ideas y conocimientos" (11).

El 55.9\% de los docentes refirieron haber publicado algún trabajo de investigación en los últimos dos años, siendo el promedio de publicaciones de 0.8 por semestre, pero debemos tener en cuenta que no existen suficientes revistas en nuestro medio para publicar un volumen de tal magnitud. Lo afirmado por los profesores es explicable por las publicaciones cortas que se hacen en los libros de resúmenes de los diversos congresos que se realizan en el año, pero que no son publicaciones en sentido estricto.

El número de publicaciones guarda relación con mayor tiempo de ejercicio docente, dedicación a la investigación y grado académico, siendo mayor la producción para los profesores principales, los que realizan investigación en forma permanente y los que tienen grado de maestro o de doctor.

El 61.9\% de nuestros docentes no está suscrito a publicaciones científicas extranjeras periódicas, proporción similar a la del médico de Lima Metropolitana en general, 73.1\% (1).

Por razones económicas, es costumbre en nuestro medio que las publicaciones se obsequien a los profesionales mientras que las publicaciones extranjeras necesariamente se hacen por suscripción previo pago del mismo. De los que se suscriben, el $66 \%$ la abandonan por razones económicas (1).

Sólo el 39.2\% de nuestros docentes realizaron algún viaje de capacitación académica en el exterior. Generalmente estos viajes son posibles por gastos propios del docente o a cuenta de otras instituciones, existiendo escasa o ninguna subvención por la universidad. 
La mitad de nuestros profesores, 52.1\%, no cuenta con un ambiente en sus centros de trabajo donde estudiar, investigar o prestar asesoría a sus alumnos y tan sólo una menor proporción cuenta con apoyo secretarial, 40.5\%, que les facilite dicha labor.

Es necesario resaltar que a pesar de lo costoso que resulta adquirir publicaciones recientes, el $88.3 \%$, utilizaba material de su propiedad y de la biblioteca central de la universidad, $54.5 \%$, siendo actualizado en su mayoría, $88.4 \%$.

El Ministerio de Salud resultó ser el mayor empleador dado que más del $90 \%$ de nuestros docentes trabajan en él. Existe un porcentaje importante de docentes, $40.8 \%$ que comparte su puesto con el de entidades privadas (clínicas preferentemente), el $17.5 \%$ enseña en otras universidades y el 5\% enseña exclusivamente en la UPCH.

En cuanto a la práctica privada en consultorio, ésta es ejercida por la mayoría de los docentes, $79.2 \%$. Los docentes que no tenían consultorio particular generalmente trabajaban para alguna otra institución.

Existe un porcentaje considerable de docentes que no son propietarios de su vivienda, $25.0 \%$, ni del consultorio particular, $53.1 \%$, siendo más notorio entre los más jóvenes.

El $13.3 \%$ de los docentes carece de carro propio, que es un medio de transporte importante en nuestro medio, más aún si se tiene en cuenta la distribución geográfica de los domicilios de los docentes.

El $17.5 \%$ de los docentes son accionistas de una clínica particular y el $41.7 \%$ de los docentes no son miembros de algún club donde puedan recrearse.

Es alta la proporción de docentes que realizó viajes recreativos al exterior, $38.1 \%$. Curiosamente esta cifra coincide con el porcentaje de profesores que viajaron por razones académicas en el mismo período, 39.2\%. Esto es explicable porque suele aprovecharse un viaje para realizar ambas actividades.

Aproximadamente la mitad de los docentes, 52.5\%, tiene otro miembro familiar que genera ingresos económicos y que es el cónyuge en la mayoría de los casos.

Son muy pocos los docentes con actividades económicas complementarias no relacionadas a la profesión de médico y/o docente, $3.3 \%$.

Como era de esperarse por las características profesionales de nuestros docentes, la actividad privada profesional representa la fuente principal de ingreso económico en el $72.5 \%$ de ellos. También es notorio el porcentaje de docentes, $20.0 \%$, cuya fuente de ingreso principal proviene de su empleo en los hospitales estatales, que, como es bien sabido les otorga una pobre remuneración.

Con respecto a los docentes de nuestra Facultad de Medicina podemos afirmar:

1. Por sus características de profesión, vivienda y acceso a bienes el promedio de nuestros docentes se ubica en el estrato socioeconómico medio de nuestra sociedad. 
2.La docencia médica que realiza resulta ser una actividad complementaria de la actividad profesional.

3.En su mayoría tienen vocación e interés por la enseñanza más no reciben las facilidades que desearían para el mejor desempeño de sus funciones.

4.No obstante la experiencia práctica como docentes, una proporción importante de ellos no tiene capacitación pedagógica .

5.La producción en lo referente a investigación y publicaciones es insuficiente.

\section{Correspondencia:}

Dr. Oscar Pamo, Universidad Peruana Cayetano Heredia. Apartado postal 5045, Lima 100, PERU.

\section{REFERENCIAS BIBLIOGRÁFICAS}

1. Alarcón J. Situación de trabajo y características socioeconómicas del médico de Lima Metropolitana (Resultados de una encuesta). Lima: C.M.P./O.P.S.; 1989.

2. Asociación Peruana de Facultades de Medicina (ASPEFAM). La autoevaluación. Análisis prospectivo de las Facultades de Medicina del Perú. 1985-1986.

3. Universidad Peruana Cayetano Heredia. Boletín Estadístico Universidad Peruana Cayetano Heredia. Lima: Archivo de la Secretaría del Decanato- Universidad Peruana Cayetano Heredia; 1986.

4.Cueto FC. Bases de la Universidad Peruana. Lima; 1970.

5.Dejo F. Situación del Docente Universitario en Lima Metropolitana. Lima: U.N.A.L.M; 1988.

6. INE. Encuesta nacional de hogares sobre medición de niveles de vida. Dirección General de Indicadores Económicos y Sociales. Lima: INE; 1990.

7. Fernández O. Situación de trabajo de médicos egresados entre los años 1981-1985 de las Facultades de Medicina de la Paz y Cochabamba. Educ.Med. y Salud 1989.p. 391401.

8. Ferreyra J. El análisis prospectivo de la Educación Médica en América Latina. Educ Med y Salud 1988; 22:279

9. La enseñanza universitaria en el ciclo básico. Resumen y conclusiones del seminario, Lima: Universidad de Lima; 1987. p. 43.

10.Lip C. El trabajo médico en el Perú. OPS y UPCH. Universidad Peruana Cayetano Heredia; 1990.

11.Mc Gregor F. Sociedad, Ley y Universidad Peruana. Lima: Fondo Editorial de la Pontificia Universidad Católica del Perú; 1988.

12. Universidad Peruana Cayetano Heredia. Libro de Presupuesto 1989. Lima:UPCH; 1989.

13. Universidad Peruana Cayetano Heredia Reglamento sobre régimen de dedicación y modalidad de trabajo del personal docente de la Universidad Peruana Cayetano Heredia, Lima: Universidad Peruana Cayetano Heredia ;1990.

14. Vasco A. Salud, Medicina y Clases Sociales. Lima: Mosca Azul Editores; 1982. p. 113-114. 